\title{
CALCULATIONS ON THE HELIUM ISOELECTRONIC SEQUENCE USING COULOMB GREEN'S FUNCTION VÁRIABLES
}

\author{
Xianqian SHI and S.M.BLINDER \\ Department of Chemistry, University of Michigan, Ann Arbor, Michigan 48109. USA
}

Received 2 September 1983

\begin{abstract}
Calculations on the ground states of the heiium isoelectronic series are carried out using variational wavefunctions of the form $\psi(x, y)$, in which $x$ and $y$ are the combinations $r_{1}+r_{2} \pm r_{12}$ occurring in the Coulomb Green's function. The results for helium are the most accurate to date using a two-variable wavefunction, accounting for $71.5 \%$ of the correlation energy.
\end{abstract}

The Coulomb Green's function is the solution under specified boundary conditions of the differential equations [1] :

$\left(\frac{1}{2} k^{2}+\frac{1}{2} \nabla_{i}^{2}+Z / r_{i}\right) G\left(r_{1}, r_{2}, k\right)=\delta\left(r_{12}\right), \quad i=1,2$.

We use atomic units, whereby $\hbar=e=\mu=1$. The sum of eqs. (1) over the two particle labels gives

$\left(k^{2}+\frac{1}{2} \nabla_{1}^{2}+\frac{1}{2} \nabla_{2}^{2}+Z / r_{1}+Z / r_{2}\right) G\left(r_{1}, r_{2}, k\right)=2 \delta\left(r_{12}\right)$,

which closely resembles the Schrödinger equation for a helium-like atom. As shown by Hostler [2] the Coulomb Green's function depends on just two variables:

$x \equiv r_{1}+r_{2}+r_{12}, \quad y \equiv r_{1}+r_{2}-r_{12}$,

rather than three - say $r_{1}, r_{2}, r_{12}-$ which would be expected on the basis of rctational invariance alone. This reduction is a consequence of the $S O(4)$ dynamical symmetry of the Coulomb problem. This higher symmetry is also connected with the existence of an additional constant of the motion, the Runge-Lenz vector.

Specifically the retarded Coulomb Green's function $G^{+}(x, y, k)$, is given by [3] :

$$
\begin{aligned}
& G^{+}(x, y, k)=-(\pi \mathrm{i} k)^{-1}(x-y)^{-1}(\partial / \partial x-\partial / \partial y) \\
& \quad \times \Gamma(1-\mathrm{i} \nu) M_{\mathrm{i} \nu}^{1 / 2}(-\mathrm{i} k y) W_{\mathrm{i} \nu}^{1 / 2}(-\mathrm{i} k x), \\
& \nu \equiv Z / k, \quad \operatorname{Im} k>0,
\end{aligned}
$$

in which $M$ and $W$ are Whittaker functions as defined by Buchholz [4].

In this note, we shall explore variational wavefunctions of the form $\psi(x, y)$ to approximate ${ }^{1} \mathrm{~S}$ states of the helium isoelectronic series. Rigorously, of course, three variables such as $r_{1}, r_{2}, r_{12}$ are required to span the functional domain for $\mathrm{S}$ states. It is of interest, nevertheless, to see how far one can go with just $x$ and $y$ dependence.

The classical work of Hylleraas [5] was based on the independent variables:

$s \equiv r_{1}+r_{2}, \quad t \equiv r_{1}-r_{2}, \quad u \equiv r_{12}$.

Since $x=s+u$ and $y=s-u$, our computations will be equivalent to those of Hylleraas with the variable $t$ absent. by

For helium-like systems, the hamiltonian is given

$H=-\frac{1}{2} \nabla_{1}^{2}-\frac{1}{2} \nabla_{2}^{2}-Z / r_{1}-Z / r_{2}+1 / r_{12}$.

For $\psi=\psi(x, y)$, we write the variational integral

$E=(\psi, H \psi) /(\psi, \psi)=(T+V) / N$;

with 


$$
\begin{aligned}
N= & \frac{1}{48} \int_{0}^{\infty} \int_{0}^{x}\left(x^{2}+4 x y+y^{2}\right)(x-y)^{2}|\psi(x, y)|^{2} \mathrm{~d} x \mathrm{~d} y, \\
T & =\frac{1}{12} \int_{0}^{\infty} \int_{0}^{x}\left[\left(x^{2}+2 x y\right)(\partial \psi / \partial x)^{2}\right. \\
& \left.+\left(y^{2}+2 x y\right)(\partial \psi / \partial y)^{2}\right](x-y)^{2} \mathrm{~d} x \mathrm{~d} y, \\
V & =-\frac{1}{4} Z \int_{0}^{\infty} \int_{0}^{x}(x+y)(x-y)^{2}|\psi(x, y)|^{2} \mathrm{~d} x \mathrm{~d} y \\
& +\frac{1}{24} \int_{0}^{\infty} \int_{0}^{x}\left(x^{2}+4 x y+y^{2}\right)(x-y)|\psi(x, y)|^{2} \mathrm{~d} x \mathrm{~d} y .
\end{aligned}
$$

Specifically, we consider linear variational functions of the form

$$
\psi(x, y)=\mathrm{e}^{-\alpha(x+y)} \sum_{m, n} C_{m, n} x^{m} y^{n}
$$

The integrals (8)-(10) are then given explicitly by

$$
\begin{aligned}
N= & \frac{1}{48} \sum_{m, n, k, l}\{[4,0]+2[3,1]-6[2,2] \\
& +2[1,3]+[0,4]\}, \\
T= & \frac{1}{12} \alpha^{2} N-\frac{1}{12} \sum_{m, n, k, l} C_{m, n} C_{k, l} \\
& \times\{2 \alpha[m([3,0]-3[1,2]+2[0,3]) \\
& +n(2[3,0]-3[2,1]+[0,3])] \\
& -m k([2,0]-3[0,2]+2[-1,3]) \\
& -n l(2[3,-1]-3[2,0]+[0,2])\}, \\
V= & \frac{1}{48} \sum_{m, n, k, l} C_{m, n} C_{k, l}\{(-12 Z+2)[3,0] \\
& +(12 Z+6[2,1]+(12 Z-6)[1,2] \\
= & +(-12 Z-2)[0,3]\} .
\end{aligned}
$$

We have introduced the notation

$$
[I, J] \equiv(m+k+I, n+l+J) \text {, }
$$

Table 1

Helium calculations with $N$-term basis

\begin{tabular}{rll}
\hline$N$ & $\alpha$ & $-E$ \\
\hline 1 & 0.84375 & 2.84765625 \\
3 & 0.905 & 2.8912315 \\
6 & 0.935 & 2.8916945 \\
10 & 0.99 & 2.8917460 \\
15 & 1.03 & 2.891750194 \\
\hline
\end{tabular}

$$
\begin{aligned}
& (m, n) \equiv \int_{0}^{\infty} \int_{0}^{x} \mathrm{e}^{-2 \alpha(x+y)} x^{m} y^{n} \mathrm{~d} x \mathrm{~d} y \\
& =\sum_{r=0}^{n} \frac{(-1)^{r} n !(m-n-r) !}{(n-r) !(2 \alpha)^{r+1}(4 \alpha)^{m+n-r+1}}+\frac{n ! m !}{(2 \alpha)^{n+m+2}}
\end{aligned}
$$

The functional form (11) trivially includes the simple exponential $\exp [-\alpha(x+y)]$, equivalent to the scaled hydrogenic product $\exp \left[-2 \alpha\left(r_{1}+r_{2}\right)\right]$. This is optimized for $\alpha=27 / 32$, giving the approximate helium ground-state energy $E=-2.84765625$ hartree. With the inclusion of sets of linear terms containing higher powers of $x$ and $y$, we obtain the results summarized in table 1 . The exponential parameter has been reoptimized for each basis set. The three-term function $(1, x, y)$ gives an optimal energy of -2.8912315 $H$. The six-term function (adding $x^{2}, x y, y^{2}$ ) gives $-2.8916504 \mathrm{H}$. The ten-term function (adding $x^{3}$,

Table 2

Coefficients for helium fifteen-term function, $\alpha=1.03$

\begin{tabular}{lr}
$C(0,0)$ & 1.000000000 \\
$C(1,0)$ & 0.265456196 \\
$C(0,1)$ & -0.175206467 \\
$C(2,0)$ & 0.017240841 \\
$C(1,1)$ & 0.080962524 \\
$C(0,2)$ & -0.041635911 \\
$C(3,0)$ & 0.002107589 \\
$C(2,1)$ & -0.008768843 \\
$C(1,2)$ & 0.001859248 \\
$C(0,3)$ & -0.000208398 \\
$C(4,0)$ & 0.000080883 \\
$C(3,1)$ & 0.001069403 \\
$C(2,2)$ & 0.000208337 \\
$C(1,3)$ & -0.000588844 \\
$C(0,4)$ & 0.000024063 \\
\hline
\end{tabular}


Table 3

Helium isoelectronic sequénce ground-state energies using fifteen-term function

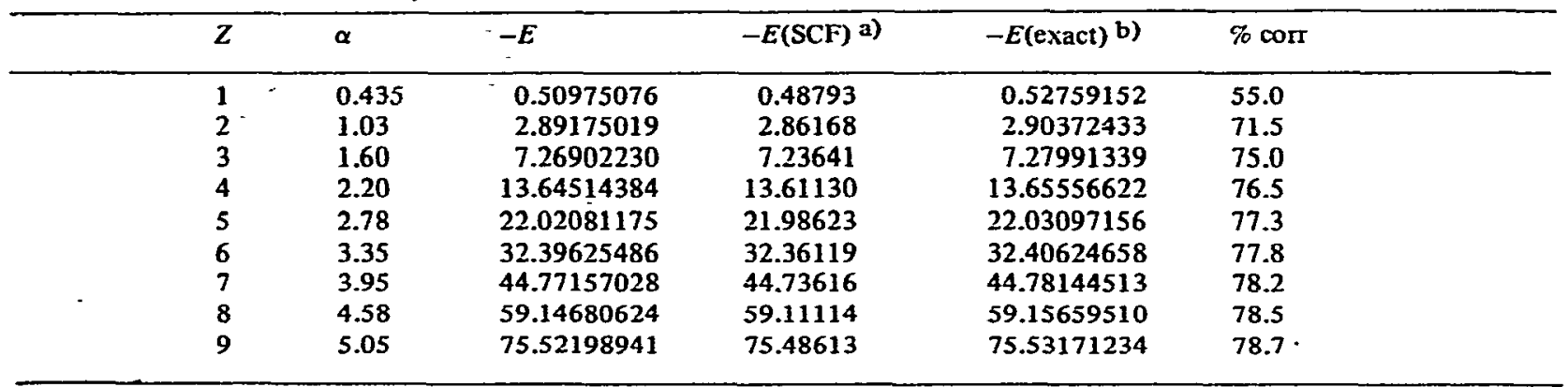

a) Ref. [7]. b) Ref. [8].

$\left.x^{2} y, x y^{2}, y^{3}\right)$ gives -2.8917460 H. Finally, with fifteen terms (adding $x^{4}, x^{3} y, x^{2} y^{2}, x y^{3}, y^{4}$ ), we ob. tain $-2.891750194 \mathrm{H}$. The coefficients are shown in table 2 . It is estimated that with additional terms the procedure would converge to an approximate energy of $-2.89175065 \mathrm{H}$. Given the exact non-relativistic energy of $-2.903724375 \mathrm{H} \mathrm{[6]}$ and the Hartree-Fock energy of $-2.86168 \mathrm{H} \mathrm{[7]}$, it is seen that our calculation accounts for $\approx 71.5 \%$ of the correlation energy of helium.

In table 3, we present analogous calculations, using the fifteen-term function, on the $1 \mathrm{~s}^{2}{ }^{1} \mathrm{~S}$ ground states of the helium isoelectronic sequence from $Z=1$ to $Z=9$. The results are compared with the self-consistent-field calculations of Roothaan and Weiss [7] and the essentially exact 13 th-order perturbation calculations of Scherr and Knight [8] . As shown in the last column of table 3 , the fraction of correlation energy captured by $\psi(x, y)$ increases to over $75 \%$ for twoelectron atoms beyond helium.

The calculations on helium-like systems described in this note are the most accurate to date based on wavefunctions dependent on just two variables.

\section{References}

[1] J. Meixner, Math. Z. 36 (1933) 677.

[2] L. Hostler, J. Math. Phys. 8 (1967) 642.

[3] L. Hostler and R.H. Pratt, Phys. Rev. Letters 10 (1963) 469; L. Hostler, J. Math. Phys. 5 (1964) 591; S.M. Blinder, Intern. J. Quantum Chem. S14 (1980) 43; J. Math. Phys. 22 (1981) 306.

[4] H. Buchholz, The confluent hypergeometric function (Springer, Berlin, 1969).

[5] E. Hylleraas, Z. Physik 48 (1928) 469; 54 (1929) 347; 65 (1930) 209; Advan. Quantum Chem. 1 (1964) 1; H.A. Bethe and E.E. Salpeter, Quantum mechanics of one- and twoelectron atoms (Academic Press, New York, 1957) pp. $146 \mathrm{ff}$.

[6] C.L. Pekeris, Phys. Rev. 115 (1959) 1216

[7] C.C.J. Roothaan and A.W. Weiss, Rev. Mod. Phys. 32 (1960) 194.

[8] C.W. Scherr and R.E. Knight, Rev. Mod. Phys. 35 (1963) 436. 